\title{
Cryptococcus cutaneous: marker of severity in disseminated infection
}

\author{
Ivan Cadena Vélez ${ }^{* 1}$, Marta Valentim ${ }^{1}$, Sara Nicolau ${ }^{1}$, Francisco Gil ${ }^{2}$, Manuela Grego ${ }^{1}$, Luis Siopa ${ }^{1}$ \\ ${ }^{1}$ Internal Medicine Department, Hospital Distrital de Santarém, Portugal \\ ${ }^{2}$ Dermatology Departament, Hospital Distrital de Santarém, Portugal
}

Received: July 11, 2019

Accepted: December 20, 2019 Online Published: February 26, 2020

DOI: $10.5430 /$ crim.v7n1p19

URL: https://doi.org/10.5430/crim.v7n1p19

\begin{abstract}
Cryptococcosis is an opportunistic fungal infection responsible for high morbidity and mortality in patients in immunosuppressive clinical conditions. The sites most affected by the hematogenous spread after the lungs are the central nervous system and the skin. The early diagnostic associated with a standard fungal therapy is important for decrease the clinical sequelae derived. We describe an unusual situation of disseminated cryptococcosis without pulmonary involvement in a patient with newly diagnosed human immunodeficiency syndrome with cutaneous lesions as initial markers of severity and precursory of cryptococcal meningitis. In this case, the authors describe a systemic approach to how an initial skin lesion is key to reach the diagnosis and perform an early treatment avoiding irreversible sequelae.
\end{abstract}

Key Words: Cryptococcus neoformans, Human immunodeficiency virus, Amphotericin B, Disseminated cryptococcosis

\section{INTRODUCTION}

Cryptococcosis is an infection caused by the encapsulated yeast, Cryptococcus neoformans, a dimorphic fungus recovered from pigeon excreta, soil, dust and human skin. After a primary infection in the lungs, the disease can disseminate via a haematogenous route to various organs, including the central nervous system and skin, in susceptible individuals. ${ }^{[1]}$ Cutaneous cryptococcal infection should be presumed to be disseminated until proven otherwise, and a search for other sites of involvement must be undertaken immediately. Most skin and soft tissue manifestations occur in the setting of disseminated disease, which is apparent in $10 \%-15 \%$ of patients with systemic cryptococcosis. ${ }^{[2]}$ Cutaneous signs are usually secondary and act as a key marker of disseminated infection. In patients with impaired cell-mediated immunity, they may be the first indication of infection, preceding the diagnosis of disseminated disease by $2-8$ months and making its recognition crucial to early treatment.

\section{Case Presentation}

A 47-year-old male patient with a history of pulmonary valvuloplasty for 15 years and a history of tobacco use, was referred to the emergency department for a headache with irradiation to the occipital region. He associated with anorexia, weight loss of $7 \mathrm{~kg}$ at 6 months, pruritic and papules lesions disseminated with 10 days of evolution. He had recently been medicated with antihistamines (hydroxyzine) and analgesics (paracetamol, metamizole) without improvement of symptoms.

At the physical examination, the patient was conscious and oriented. He was afebrile, and all other parameters were

\footnotetext{
*Correspondence: Ivan Cadena Vélez; Email: icadenavelez@ gmail.com; Address: Internal Medicine Department, Hospital Distrital de Santarém, Portugal.
}

Published by Sciedu Press 
normal and without motor or sensory deficits. The patient had multiple round, papular and nodular lesions with larger central ulcerations of the head, abdomen and the legs (see Figure 1).

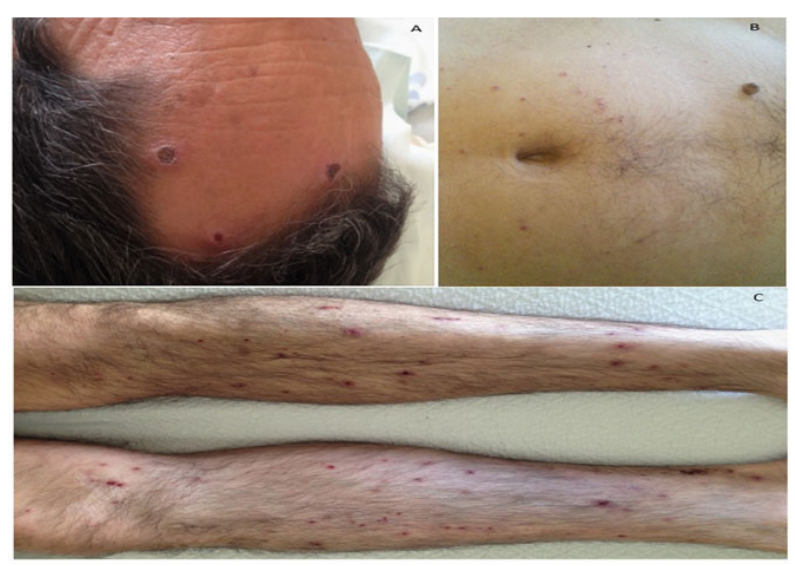

Figure 1. Cutaneous lesions of Case: A. Head: crusted and ulcerated lesions of forehead. B. Abdomen: ulcerated erythematous nodules of abdomen. C. Legs: erythematous papules and nodules of legs.

Tests performed at admission included: hemoglobin of $14.4 \mathrm{mg} / \mathrm{dl}$; leukocytes of $6,500 / \mathrm{mm}^{3}$ (75.9\% of neutrophils and $12.6 \%$ of lymphocytes; creatinine, $0.9 \mathrm{mg} / \mathrm{dl}$; urea, $37.7 \mathrm{mg} / \mathrm{dl}$; arterial blood gas without hypoxemia or respiratory insufficiency. A chest x-ray did not present any relevant alterations, and a cranioencephalic computerized tomography (CE-CT) showed: "left frontal subcortical ischemic lacuna, without evidence of lesions occupying space". Lumbar puncture was performed due to the suspicion of meningitis, and the sample was collected for cytological, bacteriological and cytochemical study. The patient was admitted for etiological clarification of the lesions.

In the course of the study of complementary diagnostic tests, the following were highlighted: Human immunodeficiency virus $1+2$ antibody (HIV $1+2$-Ac) positive, viral load (HIV) 110,000 copies, CD4 count of 27 and CD4 ratio / CD8 ratio of 0.1 . The serotypes of heterotrophic virus of hepatitis $\mathrm{B}, \mathrm{C}$ and $\mathrm{E}$, serotypes of toxoplasmosis, Interferon Gamma Release Assay (IGRA), cytomegalovirus and blood cultures were negative; from the study of cerebrospinal fluid (CSF), positivity was demonstrated for cryptococcal neoformans, confirmed later by culture of the sample. In addition, a biopsy of skin lesions revealed extensive areas of ulceration with fibrino granulocyte exudate, and in the underlying dermis, there is a marked histiocytic reaction in relation to spherical or capillary organisms compatible with cryptococcosis (see Figure 2).

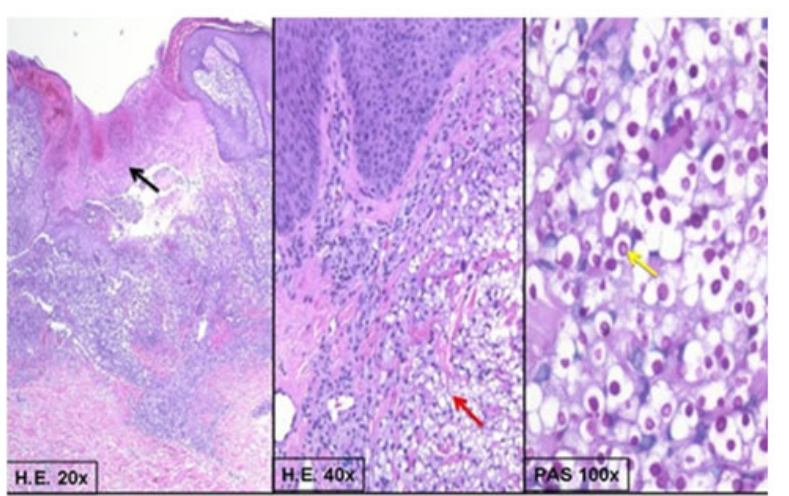

Figure 2. Histologically there is extensive ulceration with granulation tissue (black arrow); in the adjaced dermis there a mucoid mass (red arrow); where several round organisms, with a prominent PAS positive capsule (yellow arrow).

He initiated intravenous treatment with amphotericin B ( $1 \mathrm{mg} / \mathrm{kg} /$ day) plus flucytosine $(100 \mathrm{mg} / \mathrm{kg} /$ day $)$ for three weeks. Clinical symptoms, as well as disseminated skin lesions, regressed in size after the second week of antifungal treatment. At the end of the treatment, a new cultural exam of CSF was performed to evaluate the response to treatment, which was negative for Cryptococcus neoformans. The patient was discharged after completion with fluconazole in an outpatient setting (initial doses of $800 \mathrm{mg} /$ day followed by $400 \mathrm{mg} /$ day for up to 4 weeks or recovery of CD4 > 100).

\section{Discussion}

Disseminated cryptococcosis is a rare and fatal disease to occur in adults aged 20 to 50 years old, approximately $1 \%-5 \%$ of the population. Cryptococcus neoformans is one of the commonest opportunistic infections responsible for significant morbidity and mortality, primarily in immunocompromised hosts. The vast majority of infected individuals are HIV positive, transplant recipients, or have other forms of suppressed T-cell mediated immunity. ${ }^{[3,4]}$

This case shows an unusual situation of disseminated cryptococcosis without lung involvement in a patient with newlydiagnosed human immunodeficiency syndrome. Cryptococcus usually occurs in men between the ages of 30 and 50 but does not have a specific age range. It may occur in immunocompetent or immunocompromised patients, although the latter have acute and severe clinical manifestations. The severity of the clinical symptoms or signs in these patients is proportional to the number of CD4 lymphocytes at the time of diagnosis. They are more symptomatic as they present a smaller number of CD4 lower than 100/microl. Clinical manifestations such as fever, cough, dyspnea and headache occur when there is pulmonary dissemination to the central nervous system in $65 \%-94 \%$ of the HIV cases associated 
with pulmonary cryptococcosis. ${ }^{[5-7]}$ The case presented in this article shows that it does not present any respiratory symptomatology but presents nonspecific neurological manifestations associated with disseminated skin lesions.

The cutaneous lesions, in this case, cryptococcus neoformans, are usually secondary to a disseminated infection that is not limited to a single anatomical area and represent $15 \%$ of the patients with this entity whose characteristics are varied. ${ }^{\left[{ }^{8,9]}\right.}$ Patients with advanced-stage HIV infection may have molluscum-like lesions similar to the contagious molluscum diagnosis of disseminated cryptococci. Anything beyond the clinical criterion for diagnosis should be supported by direct examination with pathogen research on suspicious material such as mucocutaneous, respiratory secretions and cerebrospinal fluid (CSF) and histopathology of cutaneous lesions. ${ }^{[10]}$ Specifically, in the case presented, this patient had multiple skin in several anatomical areas associated with other systemic symptoms neurologic in the context of acute meningitis. The standard therapy or first-line induction scheme in these cases, when there is attainment of the central nervous system associated with disseminated skin lesions, is amphotericin B plus intrahospital flucytosine over a period of 2-3 weeks followed by fluconazole $400 \mathrm{mg}$ / day for 6-8 months adhering to ART (antiretroviral therapy). ${ }^{[10,11]}$ The neurologic deficit was noted initially in $37.8 \%$ of HIVnegative patients and $39.6 \%$ of HIV positive patients with cryptococcosis in one study. Neurologic sequelae in crypto-

\section{REFERENCES}

[1] Franzot SP, Salkin IF, Casadevall A. Cryptococcus neoformans var. grubii: separate varietal status for Cryptococcus neoformans serotype A isolates. J Clin Microbiol 1999; 37: 838-40. PMid:9986871. https://doi.org/10.1128/JCM.37.3.838-840.1999

[2] Anderson DJ, Schmidt C, Goodman J, et al. Cryptococcal disease presenting as cellulitis. Clin Infect Dis. 1992; 14: 666-72. PMid:1562658. https://doi.org/10.1093/clinids/14.3.6 66

[3] Mirza SA, Phelan M, Rimland D, et al. The changing epidemiology of cryptococcosis: an update from populationbased active surveillance in 2 large metropolitan areas, 1992-2000. Clin Infect Dis. 2003; 36: 789-94. PMid:12627365. https://doi.org/10.1086/368091

[4] Park BJ, Wannemuehler KA, Marston BJ, et al. Estimation of the current global burden of cryptococcal meningitis among persons living with HIV/AIDS. AIDS. 2009; 23: 525-530. PMid:19182676. https://doi.org/10.1097/QAD.0b013e328322ffac

[5] Meyohas MC, Roux P, Bollens D, et al. Pulmonary cryptococcosis: localized and disseminated infections in 27 patients with AIDS. Clin Infect Dis. 1995; 21: 628. PMid:8527555. https: //doi.org/10.1093/clinids/21.3.628

[6] Cameron ML, Bartlett JA, Gallis HA, et al. Manifestations of pulmonary cryptococcosis in patients with acquired immunodefi- coccal meningitis may include abnormal mental status (33\%), motor or cranial nerves palsies (15\%) and seizures (10\%) this neurologic deficits subsided after appropriate treatments. ${ }^{[12]}$ In this clinical case, a therapeutic follow-up was performed in the clinic with a marked resolution of symptoms and clinical signs secondary to disseminated cryptococci. It worth considering that a timely diagnosis followed by appropriate antifungal therapy will have favorable clinical and immunological results.

\section{Conclusion}

We describe a case of disseminated cryptococcosis infection in an acute clinical immunocompromised host with human immunodeficiency syndrome that initially manifested marks on the skin as the primary manifestation of neurological disease severity if having another symptomatology. The importance of presenting this rare and fatal entity with a diverse clinical presentation lies in the way in which a systematic and complex clinical approach is conducted for its timely diagnosis and the initiation of an effective treatment to avoid or reduce hidden systemic alterations that can have catastrophic consequences for the patient due inadequate treatment, such as drying them, especially neurological that can develop an acute cryptococcal meningitis in an immunocompromised patient or not.

\section{CONFLiCTS OF INTEREST Disclosure}

The authors have declared no conflicts of interest. ciency syndrome. Rev Infect Dis. 1991; 13: 64. PMid:2017634. https://doi.org/10.1093/clinids/13.1.64

[7] Wasser L, Talavera W. Pulmonary cryptococcosis in AIDS. Chest. 1987; 92: 692. PMid:3308348. https://doi.org/10.1378/ches t.92.4.692

[8] Mitchell TG, Perfect JR. Cryptococcosis in the era of AIDS100 years after the discovery of Cryptococcus neoformans. Clin Microbiol Rev. 1995; 8:515. PMid:8665468. https://doi .org/10.1128/CMR.8. 4.515

[9] Ding YG, Fang H. Edematous erythema, subcutaneous plaques, and severe pain in the lower extremities in an immunocompromised patient. JAMA. 2013; 309: 1632. PMid:23592108. https: //doi.org/10.1001/jama.2013.3740

[10] Waterston JA, Gilligan BS. Cryptococcal infections of the central nervous system: a ten year experience. Clin Exp Neurol. 1987; 23:127.

[11] Pappas PG, Perfect JR, Cloud GA, et al. Cryptococcosis in human immunodeficiency virusnegative patients in the era of effective azole therapy. Clin Infect Dis. 2001; 33: 690. PMid:11477526.https: //doi.org/10.1086/322597

[12] Neuville S, Dromer F, Morin O, et al. Primary cutaneous cryptococcos. Clin Infect Dis. 2003; 337-47. PMid:12539076. https: //doi.org/10.1086/345956 